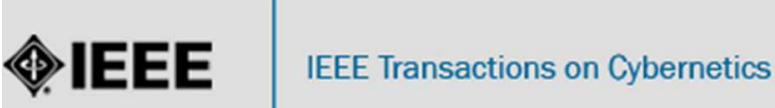

\section{Guidance-error-based Robust Fuzzy Adaptive Control for Bottom Following of a Flight-style AUV with Delayed and Saturated Control Surfaces}

\begin{tabular}{|r|l|}
\hline Journal: & IEEE Transactions on Cybernetics \\
\hline Manuscript ID & CYB-E-2018-02-0354 \\
\hline Danuscript Type: & Regular Paper \\
\hline Complete List of Authors: & $\begin{array}{l}\text { Yu, Caoyang; University of Southampton Faculty of Engineering and the } \\
\text { Environment, FSI Grounp } \\
\text { XIang, Xianbo; Huazhong University of Science and Technology, School of } \\
\text { Naval Architecture and Ocean Engineering } \\
\text { Wilson, Philip; University of Southampton Faculty of Engineering and the } \\
\text { Environment, FSI Research Group } \\
\text { Zhang, Qin; Huazhong University of Science and Technology, State Key } \\
\text { Laboratory of Digital Manufacturing, Equipment and Technology }\end{array}$ \\
\hline Keywords: & $\begin{array}{l}\text { Autonomous underwater vehicle, Actuator saturation, Delayed dynamics, } \\
\text { Fuzzy adaptive control, Robust control }\end{array}$ \\
\hline
\end{tabular}

\section{SCHOLARONE $^{\text {m }}$}




\title{
Guidance-error-based Robust Fuzzy Adaptive Control for Bottom Following of a Flight-style AUV with Delayed and Saturated Control Surfaces
}

\author{
Caoyang Yu, Xianbo Xiang, Philip A. Wilson and Qin Zhang
}

\begin{abstract}
This paper addresses the problem of robust bottom following control for a flight-style autonomous underwater vehicle (AUV) with both delayed and saturated control surfaces by using a pair of rudders. First, the time-delayed dynamics of rudders is considered, which renders a high-order nonlinear dynamics analysis and design in the model-based backstepping controller by utilizing guidance errors; Second, to overcome the shaking control behavior resulted by the model-based highorder derivative calculation, a fuzzy approximator-based modelfree controller is proposed, in order to online approximate the unknown part of the ideal backstepping architecture. In addition, the adaptive error estimation technology is resorted to compensate the system approximation error, ensuring that all the position and orientation errors of robust bottom following control tend to zero; Third, to further tackle the potential unstable control behavior from inherent saturation of control surfaces driven by rudders, an additional adaptive fuzzy compensator is introduced, in order to compensate control truncation between the unsaturated and saturation inputs. Subsequently, Lyapunov theory and Barbalat lemma are adopted to synthesize asymptotic stability of the entire bottom following control system; Finally, comparative numerical simulations among the model-based benchmark controller, the unsaturated and saturated model-free controllers are provided to illustrate adaptability and robustness of the proposed bottom following controller for a flight-style AUV with delayed and saturated control surfaces.
\end{abstract}

Index Terms-Autonomous underwater vehicle, Actuator saturation, Delayed dynamics, Fuzzy adaptive control, Robust control.

\section{INTRODUCTION}

A $\mathrm{S}$ an important branch of robotics, autonomous underwater vehicles (AUVs) are being widely used in subsea survey missions, such as seabed mapping and subsea cable

This work was supported in part by the National Natural Science Foundation of China under Grant 51579111 and Grant 51209100, in part by the Shenzhen Science and Technology Plan Project under Grant JCYJ201704I311305468, in part by the Fundamental Research Funds for the Central Universities under Grant 2017KFYXJJ005, in part by the State Key Lab Research Fund of Ocean Engineering under Grant 201504, and in part by the International Exchanges of the UK Royal Society under Grant IE161588. (Corresponding author: Xianbo Xiang)

C. Yu and X. Xiang are with the School of Naval Architecture and Ocean Engineering, Huazhong University of Science and Technology, Wuhan 430074, China. (E-mail: ycyhust@hust.edu.cn; xbxiang@hust.edu.cn)

X. Xiang is also with the Shenzhen Huazhong University of Science and Technology Research Institute, Shenzhen 518057, China.

C. Yu and P. A. Wilson are with the Faculty of Engineering and the Environment, University of Southampton, Southampton SO16 7QF, UK. (Email: C.Yu@soton.ac.uk; Philip.Wilson@soton.ac.uk)

Q. Zhang is with the State Key Laboratory of Digital Manufacturing, Equipment and Technology, Huazhong University of Science and Technology, Wuhan 430074, China. (E-mail: qin.zhang@hust.edu.cn) inspection [1]-[5]. This type of survey-style AUV usually has a torpedo-shaped body that is equipped with a stern propeller and two pairs of control surfaces driven by rudders, which suit the long-range flight-style operation at high speed [6].

Since control surfaces cannot independently generate the heave and sway forces, the above flight-style AUV is seen as a under-actuated system. The under-actuated configuration means there are more degrees of freedom to be controlled than the number of control input, which makes the controller design difficult. Taking the vertical-plane path following mission as an example, the horizontal control surface is adaptively adjusted to reduce the pitch and heave errors simultaneously. In order to address this under-actuated problem, a virtual target point is usually introduced on the desired path to add a control degreeof-freedom, and then a line-of-sight guidance law built on this target point is designed to assist the AUV to reduce the pitch and heave errors or the yaw and sway errors by only using a pair of control surfaces [7]-[10].

In addition to the under-actuated problem for a flightstyle AUV, the external dynamics can change considerably with the changes in water layers and external environmental disturbances, such as wind velocity and ocean currents [11][14]. These external dynamics with the body hydrodynamic coefficients are normally difficult to measure or predict accurately, which renders the model-based controller ineffective. To address the problem of system uncertainties caused by fully or partly unknown dynamics model, some advanced intelligent controllers have been designed over the past few decades [15]. More precisely, in [16], the time delay control was employed by an underwater inspection robot that is used to track a trapezoid trajectory for nuclear reactor internal inspection. In [17], the RISE-based controller was applied to an AUV to reject system uncertainties in the helical trajectory tracking control. In [18], the VideoRay PRO ROV was equipped with a model-free control system that can achieve trajectory tracking with prescribed performance in the presence of internal and external uncertainties.

In addition, fuzzy logic control has been widely applied to motion control of robots with system uncertainties [19]-[21]. For instance, the classic fuzzy logic was adopted to adjust control gains of a low-layer PID dynamics controller, in order to improve the system robustness [20]. An adaptive fuzzy controller was designed to deal with trajectory tracking problem of an autonomous surface vehicle with complex uncertainties [21]. Owing to the strong learning and approximation abilities, adaptive neural network controllers have been playing an 
important role in maneuvering and control of autonomous marine vehicles [22], [23].

Yet, the above research does not consider actuator saturation of marine vehicles, which means that control input acting on the vehicle is assumed to be unsaturated. In fact, actuator saturation exists in the practical vehicle system and maybe cause performance degradation even instability in the closedloop response [24]-[26]. To solve the problem of actuator saturation, a slack variable was introduced to transform the saturated fin angle control problem into the unsaturated problem, and then a state-dependent Riccati equation-based robust controller was designed for fixed-depth tracking of the REMUS AUV [27]. In [28], a nonlinear model predictive control-based tracking controller was designed so that the entire AUV closed-loop stability can be guaranteed in the presence of actuator saturation. In [29], a saturated neural adaptive robust tracking controller was designed by using generalized saturation functions to reduce the risk of actuator saturations and improve the transient performance of the trajectory tracking control system. In [30], direct adaptive fuzzy control was adopted to approximate the difference between the unsaturated and constrained control laws. In [31], a Gaussian error function-based continuous differentiable asymmetric saturation model was employed to handle the effect of nonsmooth asymmetric saturation nonlinearities.

Note that the above research does not consider time-delayed actuator dynamics, which means that the actual control output is assumed to be equal to the given command. Actually, there exists an inherent time delay between input and response [32]. In [33], a state feedback controller that is locally optimal near the origin and globally inverse optimal was derived for path following control of an over-actuated marine craft with actuator dynamics. Simulation results showed that thrust outputs with actuator dynamics are smoother than that without actuator dynamics.

To the best knowledge of the authors, there is no work on the model-free control design of a flight-style AUV with both delayed and saturated dynamics. In this paper, the objective is to address the problem of robust bottom following control of a flight-style AUV with unknown hydrodynamic coefficients, time-varying environmental disturbances, delayed rudder dynamics and saturated control surfaces. As shown in Fig. 1, three controllers including the model-based backstepping controller, the model-free unsaturated adaptive controller and the model-free saturated adaptive controller are successively derived, along with comparative numerical simulations to clearly explain the challenges of the posed robust bottom following problem.

The corresponding main contributions are summarized as follows:

(1) First, considering the time-delayed dynamics of rudders in the AUV dynamics model, a model-based high-order backstepping controller by utilizing guidance errors is designed for the second-order nonlinear dynamics system. This backstepping controller also plays as a benchmark for model-free unsaturated and saturated controllers, and meanwhile provides an unified control architecture for the investigated bottom following problem;

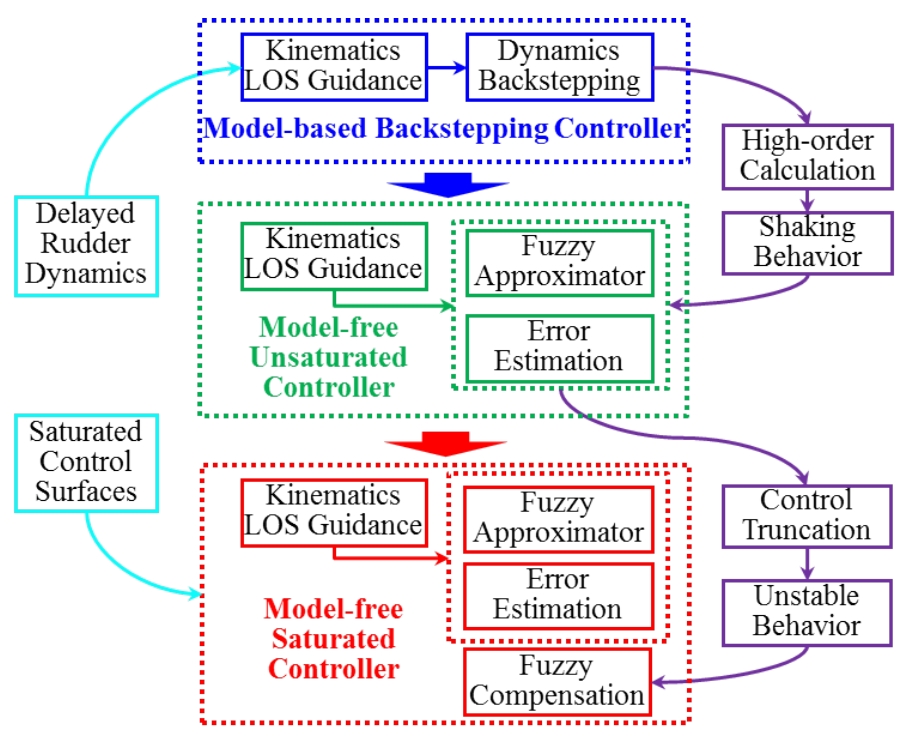

Fig. 1. Relationship among the model-based backstepping controller, the model-free unsaturated and saturated controllers

(2) Second, to overcome the shaking control behavior resulted by the model-based high-order derivative calculation, a fuzzy approximator-based model-free control law is proposed to online approximate the unknown part of the unified control architecture, which also relaxes the dependence on the accurate AUV hydrodynamic coefficients and environmental disturbances. The corresponding fuzzy approximation error is compensated by the adaptive estimation technology, ensuring that all the position and orientation errors of robust bottom following control converge to zero;

(3)Third, to further tackle the potential unstable control behavior resulted by control surface saturation, an additional adaptive fuzzy control law is resorted to compensate negative effects of control input truncation between the unsaturated and saturated control commands in the sense of system stability. Subsequently, asymptotic stability of the entire bottom following control system is formally synthesized by combining Lyapunov theory with Barbalat lemma.

The rest of this paper is organized as follows. Section II describes mathematical models of the flight-style AUV and the bottom following problem. In section III, the ideal model-based backstepping controller, the model-free unsaturated adaptive controller and the model-free saturated adaptive controller are designed, respectively. Comparative numerical results among the model-based benchmark controller, the unsaturated and saturated model-free controllers are given in section IV. Conclusions and future work are summarized in section $\mathrm{V}$.

\section{Mathematic Modeling}

This section introduces mathematic models of a flight-style AUV and the bottom following control mission. The timedelayed dynamics of rudders and the inherent saturation of control surfaces driven by rudders are included in mathematic models, which increases the order and nonlinearity of the AUV 
dynamics system as well as the complexity of the model-based controller design. Subsequently, the bottom following mission of a flight-style AUV is introduced and formulated.

\section{A. AUV Model}

Let $(x, z, \theta)$ be the vertical-plane position and pitch angle of a flight-style AUV in the earth-fixed inertial frame, and let $(u, w, q)$ be its surge, heave, and pitch velocities in the body-fixed frame. Then, the vertical-plane kinematics model that translates the body-fixed velocities into the earth-fixed velocities, can be described as follows:

$$
\begin{aligned}
& \dot{x}=u \cos (\theta)+w \sin (\theta) \\
& \dot{z}=-u \sin (\theta)+w \cos (\theta) \\
& \dot{\theta}=q
\end{aligned}
$$

Define $U=\sqrt{u^{2}+w^{2}}, \alpha=\arctan \left(\frac{w}{u}\right)$, and $v=\theta-\alpha$ as the resultant velocity, angle of attack, and elevation angle of the AUV, respectively [9], [30]. Then, the kinematics model in (1) can be rewritten as,

$$
\begin{aligned}
& \dot{x}=U \cos (v) \\
& \dot{z}=-U \sin (v) \\
& \dot{v}=q-\dot{\alpha}
\end{aligned}
$$

On the other hand, the AUV dynamics model for a flightstyle AUV in the vertical plane can be expanded as [33],

$$
\begin{aligned}
\dot{u} & =f_{u}+g_{u} n^{2} \\
\dot{w} & =f_{w} \\
\dot{q} & =f_{q}+g_{q} \delta \\
\delta_{c} & =\lambda \dot{\delta}+\delta
\end{aligned}
$$

where $f_{u}=\left[-(W-B) \sin (\theta)+X_{u|u|} u|u|+X_{q q} q^{2}+\left(X_{w q}-\right.\right.$ $\left.m) w q+\tau_{E u}\right] /\left(m-X_{\dot{u}}\right), g_{u}=\rho D^{4} K(1-t) /\left(m-X_{\dot{u}}\right), f_{w}=$ $\left[m z_{G} q^{2}+m u q+Z_{u q} u q+Z_{u w} u w+Z_{w|w|} w|w|+Z_{q|q|} q|q|+\right.$ $\left.(W-B) \cos (\theta)+\tau_{E w}\right] /\left(m-Z_{\dot{w}}\right), f_{q}=\left[-m x_{G} u q-m z_{G} w q\right.$ $+M_{u q} u q+M_{u w} u w+M_{w|w|} w|w|+M_{q|q|} q|q|-x_{G} W \cos (\theta)$ $\left.-z_{G} W \sin (\theta)+\tau_{E q}\right] /\left(I_{y y}-M_{\dot{q}}\right)$, and $g_{q}=M_{u u \delta} u^{2} /\left(I_{y y}-\right.$ $M_{\dot{q}}$ ) are compressed hydrodynamic coefficients originating from the inertia, Coriolis, damping, gravity, and environmental disturbance terms. $n$ is the propeller velocity measured in rev/min. $\delta$ and $\delta_{c}$ are the actual control surface deflection and the deflection command of the flight-style AUV, respectively. $\lambda$ denotes the time delay between them.

Remark 1 In the dynamics model (3), the delayed dynamics model of actuators including the stern propeller and the vertical control surface are introduced. Especially, the time delay between the actual control surface deflection and its command is considered because the stern control surface is usually driven by a stepper motor.

Remark 2 For any marine vehicle, there exists inherent actuator saturation. Therefore, the propeller velocity and stern control surface deflection of the flight-style AUV should be bounded, namely $|n| \leq n_{\max }$ and $\left|\delta_{c}\right| \leq \delta_{c, \max }$ where $n_{\max }$ and $\delta_{c, \max }$ are known positive constants.

Assumption 1 [13] The environmental disturbances acting on the AUV are assumed to be bounded but unknown, namely $\left|\tau_{E u}\right| \leq \tau_{E u, \max },\left|\tau_{E w}\right| \leq \tau_{E w, \max }$ and $\left|\tau_{E q}\right| \leq \tau_{E q \text {,max }}$

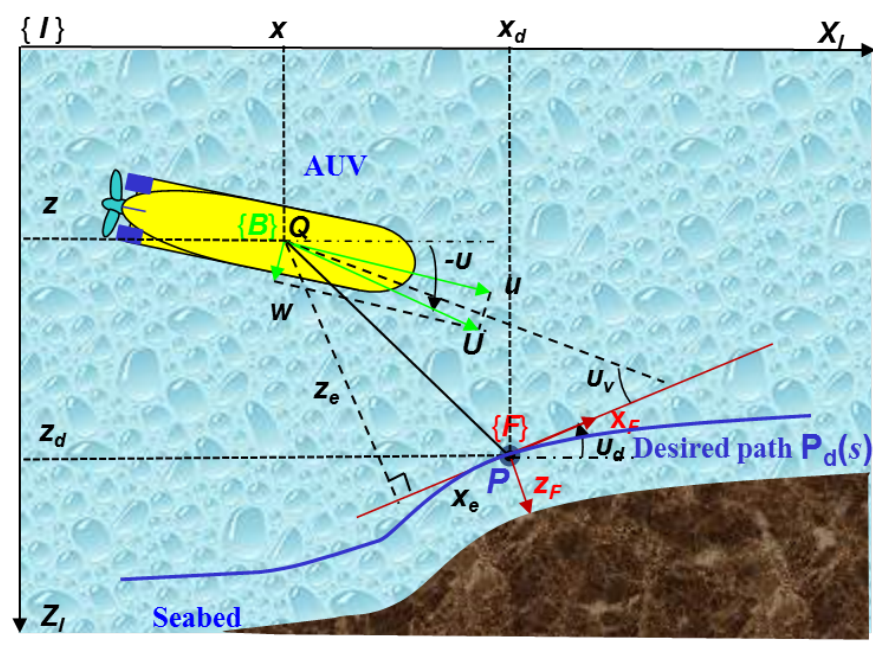

Fig. 2. Bottom following of a flight-style AUV

where $\tau_{E u, \max }, \tau_{E w, \max }$ and $\tau_{E q, \max }$ are unknown positive constants.

Assumption 2 [34] The surge velocity $u$ is decoupled from the other degrees of freedom and is assumed to remain constant during the bottom following mission, which leads to that $g_{q}$ is a negative constant by considering $M_{u u \delta}<0$, $I_{y y}>0$ and $M_{\dot{q}}<0$. Further, the last two equations in (3) can be rewritten as,

$$
\lambda \ddot{q}+\dot{q}-\lambda \dot{f}_{q}-f_{q}=g_{q} \delta_{c}
$$

Remark 3 Under assumption 2, the dynamics model in (4) becomes a second-order nonlinear system relative to the original first-order dynamics system in (3). Therefore, the stern control surface deflection command is also related to the second-order pitch angular velocity $\ddot{q}$ and the first-order heave linear velocity $\dot{w}$, which makes the model-based controller design more complex.

\section{B. Bottom Following Mission}

In the bottom following mission, the AUV is usually required to maintain a constant height over the subsea bottom so that the position and orientation errors between the AUV and the desired path over the bottom tend to zero. As shown in Fig. 2, assume that the fixed-height path to be tracked by the flight-style AUV is parameterized by $p_{d}(s)=\left[x_{d}(s), z_{d}(s)\right]$. Similar to the AUV kinematic model, the tangential angle of the point $P\left(x_{d}(s), z_{d}(s)\right)$ on this path is given by,

$$
v_{d}=-\arctan \left(\frac{z_{d}^{\prime}}{x_{d}^{\prime}}\right)
$$

with $x_{d}^{\prime}=\frac{\partial x_{d}(s)}{\partial s}$ and $z_{d}^{\prime}=\frac{\partial z_{d}(s)}{\partial s}$, and its derivative is,

$$
\dot{v}_{d}=\kappa \dot{s} \sqrt{x_{d}^{\prime 2}+z_{d}^{\prime 2}}
$$

where $\kappa$ represents the curvature of the point $P$ [35]. 


\section{Control Design}

This section describes three different controllers including the ideal model-based backstepping controller, the modelfree unsaturated adaptive controller and the saturated adaptive controller. The first backstepping controller depends on the accurate AUV hydrodynamic coefficients and environmental disturbance model, but provides as a benchmark an unified control architecture for the next two controllers. Under the above control architecture, the subsequent unsaturated adaptive controller employs adaptive fuzzy approximator and error estimation technology to relax the dependence on the AUV dynamics model, which also avoids the complex secondorder derivative calculation and the resulted shaking control behavior in the backstepping control. Finally, the saturated adaptive controller is proposed to reduce negative effects of control input truncation in the unsaturated adaptive controller and guarantee the asymptotic stability despite of the bounded control surface deflection command.

\section{A. Model-based Backstepping Controller with Delayed Con- trol Surfaces}

In this subsection, a model-based backstepping controller is designed for robust bottom following control of a flight-style AUV with delayed and saturated control surface, as shown in Fig. 3. After building the tracking errors in the path frame, the line-of-sight-based guidance is resorted to construct the virtual velocity law of the target point on the path and the reference pitch angular velocity of the AUV. Assume that the accurate hydrodynamic coefficients can be solved by using the CFD simulation or the towing experiment method, and the environmental disturbances are also measured online. Then, a backstepping-based control law is proposed to complete the robust bottom following mission. The following are main results of this model-based backstepping controller.

The control objective of the bottom following mission is to let the position and orientation errors between the AUV and the desired path over the bottom be zero. As shown in Fig. 2, the tracking errors are built in the path frame that uses the tangent of the point $P$ as the $x$-axis direction and the normal as $z$-axis direction as follows:

$$
\begin{aligned}
x_{e} & =\left(x-x_{d}\right) \cos \left(v_{d}\right)-\left(z-z_{d}\right) \sin \left(v_{d}\right) \\
z_{e} & =\left(x-x_{d}\right) \sin \left(v_{d}\right)+\left(z-z_{d}\right) \cos \left(v_{d}\right) \\
v_{e} & =v-v_{d}
\end{aligned}
$$

In terms of (2), (5) and (6), differentiating (7) yields,

$$
\begin{aligned}
& \dot{x}_{e}=-\dot{s}\left(1+\kappa z_{e}\right) \sqrt{x_{d}^{\prime 2}+z_{d}^{\prime 2}}+U \cos \left(v_{e}\right) \\
& \dot{z}_{e}=\kappa x_{e} \dot{s} \sqrt{x_{d}^{\prime 2}+z_{d}^{\prime 2}}-U \sin \left(v_{e}\right) \\
& \dot{v}_{e}=q-\dot{\alpha}-\kappa \dot{s} \sqrt{x_{d}^{\prime 2}+z_{d}^{\prime 2}}
\end{aligned}
$$

Theorem 1 Consider a flight-style AUV with the kinematics equations in (1) and the dynamics equations in (3), and assume that the surge velocity $u$ remains constant. Let the stern control surface deflection command $\delta_{c}$ and the virtual velocity control law $\dot{s}$ be designed as,

$$
\delta_{c}=\frac{1}{g_{q}}\left(\lambda \ddot{q}-\lambda \dot{f}_{q}-f_{q}+\dot{q}_{d}\right)+\frac{1}{g_{q}}\left(-k_{3} q_{e}-v_{e}+v_{v}\right)
$$

and

$$
\dot{s}=\frac{k_{1} x_{e}+U \cos \left(v_{e}\right)}{\sqrt{x_{d}^{\prime 2}+z_{d}^{\prime 2}}}
$$

where $k_{1}>0$ and $k_{3}>0$, the guidance pitch velocity $q_{d}$ is updated by,

$$
\begin{aligned}
q_{d}= & \dot{v}_{v}+z_{e} U \frac{\sin \left(v_{e}\right)-\sin \left(v_{v}\right)}{v_{e}-v_{v}}-k_{2}\left(v_{e}-v_{v}\right)+\dot{\alpha} \\
& +\kappa \dot{s} \sqrt{x_{d}^{\prime 2}+z_{d}^{\prime 2}}
\end{aligned}
$$

with a positive constant $k_{2}$, and the corresponding pitch velocity guidance error is,

$$
q_{e}=q-q_{d}
$$

In addition, the time-varying line-of-sight guidance angle is given by,

$$
v_{v}=\arctan \left(\frac{z_{e}}{k U}\right)
$$

with a positive constant $k$. Then, the equilibrium point $\left(x_{e}, z_{e}, v_{e}\right)=(0,0,0)$ of the entire bottom following control system is globally asymptotically stable.

Proof Define the radically unbounded and positive definite Lyapunov candidate function $V_{1}$ as follows:

$$
V_{1}=\frac{1}{2}\left[x_{e}^{2}+z_{e}^{2}+\left(v_{e}-v_{v}\right)^{2}+q_{e}^{2}\right]
$$

Substituting (9) into (4) yields,

$$
\dot{q}-\dot{q}_{d}=-k_{3} q_{e}-v_{e}+v_{v}
$$

Combining (8) with (11) leads to,

$$
\dot{v}_{e}-\dot{v}_{v}=q_{e}+z_{e} U \frac{\sin \left(v_{e}\right)-\sin \left(v_{v}\right)}{v_{e}-v_{v}}-k_{2}\left(v_{e}-v_{v}\right)
$$

Resorting to the error dynamics (8), (15) and (16), the derivative of $V_{1}$ is,

$$
\begin{aligned}
\dot{V}_{1}= & x_{e}\left(-\dot{s}\left(1+\kappa z_{e}\right) \sqrt{x_{d}^{\prime 2}+z_{d}^{\prime 2}}+U \cos \left(v_{e}\right)\right) \\
& +z_{e}\left(\kappa x_{e} \dot{s} \sqrt{x_{d}^{\prime 2}+z_{d}^{\prime 2}}-U \sin \left(v_{e}\right)\right) \\
& +\left(v_{e}-v_{v}\right)\left(q_{e}+z_{e} U \frac{\sin \left(v_{e}\right)-\sin \left(v_{v}\right)}{v_{e}-v_{v}}-k_{2}\left(v_{e}-v_{v}\right)\right) \\
& +q_{e}\left(-k_{3} q_{e}-v_{e}+v_{v}\right) \\
= & -x_{e}\left(\dot{s} \sqrt{x_{d}^{\prime 2}+z_{d}^{\prime 2}}-U \cos \left(v_{v}\right)\right)-z_{e} U \sin \left(v_{e}\right) \\
& -k_{2}\left(v_{e}-v_{v}\right)^{2}-k_{3} q_{e}^{2} \\
= & -k_{1} x_{e}^{2}-\frac{U}{\sqrt{z_{e}^{2}+k^{2} U^{2}}} z_{e}^{2}-k_{2}\left(v_{e}-v_{v}\right)^{2}-k_{3} q_{e}^{2}
\end{aligned}
$$

which implies that the derivative of $V_{1}$ is negative definite. It is concluded that $\lim _{t \rightarrow \infty}\left(x_{e}, z_{e}, v_{e}\right)=(0,0,0)$ based on the Lyapunov's second method for stability. This concludes the proof of theorem 1.

Remark 4 It can be seen from (9) that the second-order derivative of velocities is required but cannot be unmeasured by sensors. In addition, the first-order and second-order derivatives are prone to induce the shaking signal [36]. On the other 


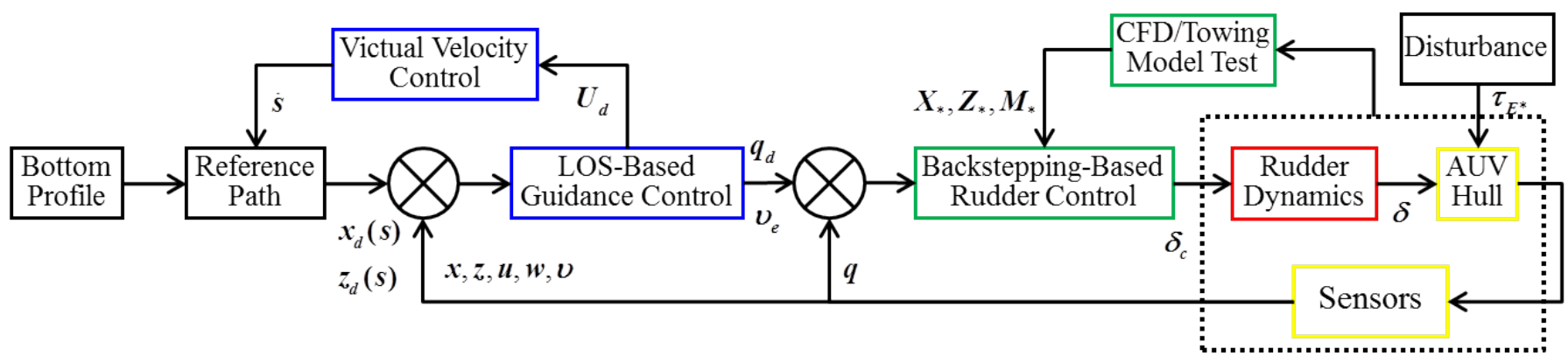

Fig. 3. Block diagram of the model-based backstepping bottom following control system

hand, it is difficult to obtain both the accurate hydrodynamic coefficients and the environmental disturbance model by using the simulation and experiment methods. Therefore, using the above ideal model-based backstepping control law in (9) is impossible in real application. The following is a model-free adaptive control law with the same control architecture.

\section{B. Model-free Adaptive Controller with Delayed and Unsatu- rated Control Surfaces}

Recall (9) and let $\delta_{0}=\frac{1}{g_{q}}\left(\lambda \ddot{q}-\lambda \dot{f}_{q}-f_{q}+\dot{q}_{d}\right), \delta_{1}=\frac{1}{g_{q}}$ and $A=-k_{3} q_{e}-v_{e}+v_{v}$. Then, the model-based backstepping control law in (9) can be expressed in a simplified form:

$$
\delta_{c}=\delta_{0}+\delta_{1} A
$$

To address the problem of system uncertainties and unmeasured derivatives, a model-free adaptive controller is proposed based on fuzzy universal approximation theorem and adaptive error estimation technology, as shown in Fig. 4. The universal approximation theorem is used to approximate the ideal rudder control law, which deals with fully unknown dynamics model uncertainties and unmeasured second-order derivatives. Then, the adaptive estimation law is further designed to tackle the fuzzy approximation error, which guarantees the asymptotic stability. The following are main results of this model-free unsaturated adaptive controller.

First, the following universal approximation theorem is reviewed:

Lemma 1 [37], [38] For a real continuous function $u(e)$ whose analytic expression is unknown, there exists an optimal fuzzy control system such that,

$$
u(e)=\varpi^{* \top} \boldsymbol{\xi}+\varepsilon
$$

where $\varpi^{*}=\left[\varpi_{1}^{*}, \varpi_{2}^{*}, \cdots, \varpi_{n}^{*}\right]^{\top}$ is a parameter vector, $\boldsymbol{\xi}=\left[\xi_{1}, \xi_{2}, \cdots, \xi_{n}\right]^{\top}$ is a regressive vector, and $\varepsilon$ is the fuzzy approximation error.

According to Lemma 1, there is,

$$
\delta_{0}+\delta_{1} A=\varpi_{0}^{* \top} \boldsymbol{\xi}_{0}+\varpi_{1}^{* \top} \boldsymbol{\xi}_{1} A+\epsilon_{01}
$$

Assumption 3 [21] The system approximation error $\epsilon_{01}$ satisfies $\left|\epsilon_{01}\right| \leq E$ with an unknown upper bound $E>0$.

Theorem 2 Consider a flight-style AUV with the kinematics equations in (1) and the dynamics equations in (3), and assume that the surge velocity $u$ remains constant. Let the virtual control law $\dot{s}$ be updated by (10) and the stern control surface deflection command $\delta_{c}$ be updated by,

$$
\delta_{c}=\hat{\varpi}_{0}^{T} \boldsymbol{\xi}_{0}+\hat{\boldsymbol{\varpi}}_{1}^{T} \boldsymbol{\xi}_{1} A+\hat{E} \operatorname{sgn}\left(q_{e}\right)
$$

where $\hat{\varpi}_{0}, \hat{\varpi}_{1}$, and $\hat{E}$ are adaptive estimations of $\varpi_{0}^{*}, \varpi_{1}^{*}$, and $E$, respectively. They are governed by,

$$
\begin{aligned}
\dot{\hat{\boldsymbol{\varpi}}}_{0} & =\eta_{0}^{-1} q_{e} \boldsymbol{\xi}_{0} \\
\dot{\hat{\boldsymbol{\varpi}}}_{1} & =\eta_{1}^{-1} q_{e} \boldsymbol{\xi}_{1} A \\
\dot{\hat{E}} & =\eta_{2}^{-1}\left|q_{e}\right|
\end{aligned}
$$

with $\eta_{0}>0, \eta_{1}>0$ and $\eta_{2}>0$. Then, the equilibrium point $\left(x_{e}, z_{e}, v_{e}\right)=(0,0,0)$ of the entire bottom following control system is stable.

Proof Define a new radically unbounded and positive definite Lyapunov candidate function as follows:

$$
V_{2}=V_{1}-\frac{1}{2} g_{q}\left(\eta_{0} \tilde{\varpi}_{0}^{T} \tilde{\varpi}_{0}+\eta_{1} \tilde{\varpi}_{1}^{T} \tilde{\varpi}_{1}+\eta_{2} \tilde{E}^{2}\right)
$$

where $\tilde{\varpi}_{0}=\hat{\varpi}_{0}-\varpi_{0}^{*}, \tilde{\varpi}_{1}=\hat{\varpi}_{1}-\varpi_{1}^{*}$, and $\tilde{E}=\hat{E}-E$. Note that the positive definite function is guaranteed by $g_{q}<0$ as mentioned in assumption 2.

Substituting (20) and (21) into (4) yields,

$$
\begin{aligned}
\dot{q}-\dot{q}_{d}= & -k_{3} q_{e}-v_{e}+v_{v} \\
& +g_{q}\left(\tilde{\boldsymbol{\varpi}}_{0}^{T} \boldsymbol{\xi}_{0}+\tilde{\boldsymbol{\varpi}}_{1}^{T} \boldsymbol{\xi}_{1} A+\hat{E} \operatorname{sgn}\left(q_{e}\right)-\epsilon_{01}\right)
\end{aligned}
$$

Resorting to the error dynamics (8), (16) and (24), the 


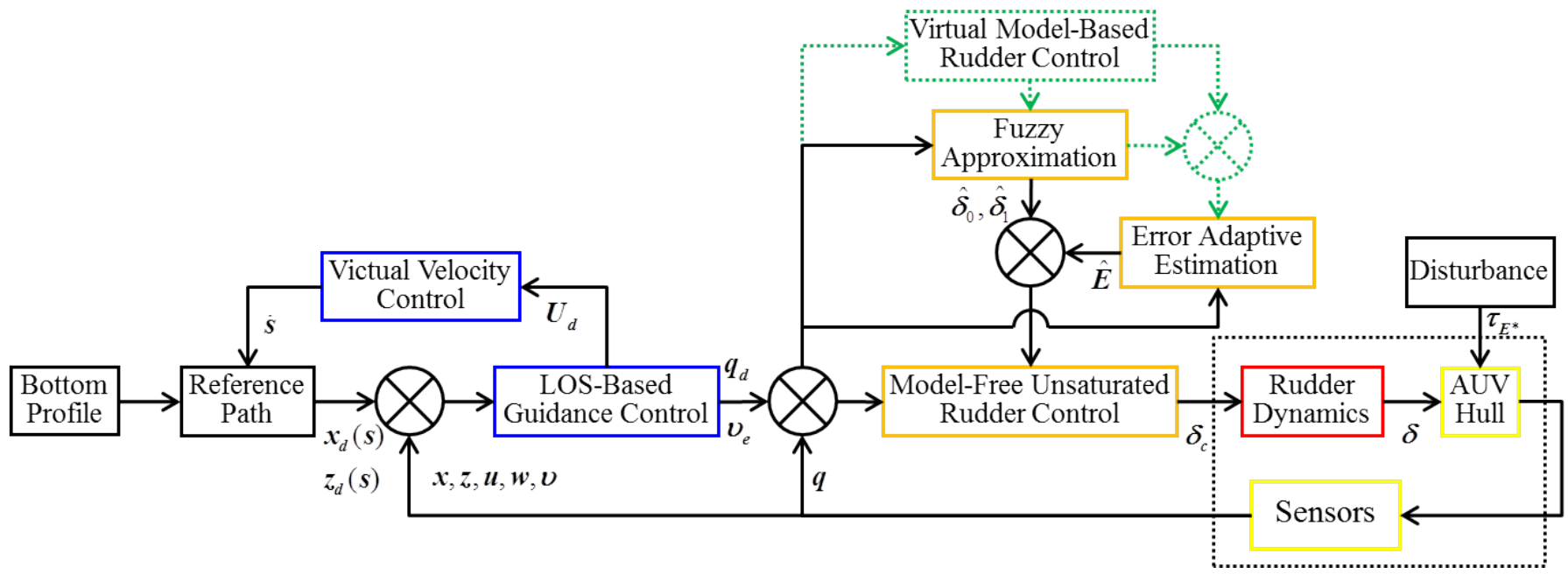

Fig. 4. Block diagram of the model-free unsaturated bottom following control system

derivative of $V_{2}$ is,

$$
\begin{aligned}
\dot{V}_{2}= & -k_{1} x_{e}^{2}-\frac{U}{\sqrt{z_{e}^{2}+k^{2} U^{2}}} z_{e}^{2}-k_{2}\left(v_{e}-v_{v}\right)^{2}-k_{3} q_{e}^{2} \\
& +q_{e} g_{q}\left(\tilde{\varpi}_{0}^{T} \boldsymbol{\xi}_{0}+\tilde{\varpi}_{1}^{T} \boldsymbol{\xi}_{1} A+\hat{E} \operatorname{sgn}\left(q_{e}\right)-\epsilon_{01}\right) \\
& -g_{q} \eta_{0} \tilde{\varpi}_{0}^{T} \dot{\hat{\varpi}}_{0}-g_{q} \eta_{1} \tilde{\varpi}_{1}^{T} \dot{\hat{\varpi}}_{1}-g_{q} \eta_{2} \dot{\hat{E}}(\hat{E}-E) \\
= & -k_{1} x_{e}^{2}-\frac{U}{\sqrt{z_{e}^{2}+k^{2} U^{2}}} z_{e}^{2}-k_{2}\left(v_{e}-v_{v}\right)^{2}-k_{3} q_{e}^{2} \\
& +g_{q} \tilde{\varpi}_{0}^{T}\left(q_{e} \boldsymbol{\xi}_{0}-\eta_{0} \dot{\boldsymbol{\varpi}}_{0}\right)+g_{q} \tilde{\varpi}_{1}^{T}\left(q_{e} \boldsymbol{\xi}_{1} A-\eta_{1} \dot{\hat{\varpi}}_{1}\right) \\
& +g_{q} \hat{E}\left(\left|q_{e}\right|-\eta_{2} \dot{\hat{E}}\right)-q_{e} g_{q} \epsilon_{01}+g_{q} E \eta_{2} \dot{\hat{E}} \\
= & -k_{1} x_{e}^{2}-\frac{U}{\sqrt{z_{e}^{2}+k^{2} U^{2}}} z_{e}^{2}-k_{2}\left(v_{e}-v_{v}\right)^{2}-k_{3} q_{e}^{2} \\
& +g_{q}\left(E\left|q_{e}\right|-q_{e} \epsilon_{01}\right) \\
\leq & -k_{1} x_{e}^{2}-\frac{U}{\sqrt{z_{e}^{2}+k^{2} U^{2}}} z_{e}^{2}-k_{2}\left(v_{e}-v_{v}\right)^{2}-k_{3} q_{e}^{2}
\end{aligned}
$$

Assumption 4 [30] Assume that the depth error $z_{e}$ is bounded and the resultant velocity $U$ of the vehicle keeps positive. Namely, $\left|z_{e}\right| \leq z_{e, \max }$ and $U \geq U_{\min }>0$ where $z_{e, \max }$ is a positive constant.

Under Assumption 4, there exists a positive constant $k_{0}$ such that $k_{0}=\frac{U_{\min }}{\sqrt{z_{e, \text { max }}^{2}+k^{2} U_{\min }^{2}}}$. Then, $-k^{-1} \leq-\frac{U}{\sqrt{z_{e}^{2}+k^{2} U^{2}}} \leq$ $-k_{0}$. By choosing $k_{\min }=2 \min \left\{k_{0}, k_{1}, k_{2}, k_{3}\right\},(25)$ can be rewritten as,

$$
\dot{V}_{2} \leq-k_{\min } V_{1}
$$

which implies that $\dot{V}_{2}$ is a negative semi-definite function and $V_{2}(t)$ is non-increasing. Therefore, for all $t>0, V_{2}(t)$ is bounded because $V_{2}(0)$ is bounded.

Define the term,

$$
P(t)=k_{\min } V_{1} \leq-\dot{V}_{2}
$$

Since $V_{2}(0)$ and $V_{2}(\infty)$ are bounded, it can be concluded that,

$$
\int_{0}^{\infty} P(t) d t \leq V_{2}(0)-V_{2}(\infty)<\infty
$$

Moreover, $V_{2}(t)$ is bounded implies that $x_{e}, z_{e}, q_{e}, v_{e}$, $\left\|\tilde{\varpi}_{0}\right\|,\left\|\tilde{\varpi}_{1}\right\|$ and $\tilde{E}$ are also bounded. Here, $\|\cdot\|$ denotes the Euclidean norm of a vector. Further, $\hat{E}$ and $A$ are bounded. In addition, there are $\left\|\boldsymbol{\xi}_{0}\right\| \leq 1$ and $\left\|\boldsymbol{\xi}_{1}\right\| \leq 1$. Therefore, there is,

$$
\begin{aligned}
\dot{P}(t) & =k_{\min } \dot{V}_{1} \\
& =k_{\min }\left\{-k_{1} x_{e}^{2}-\frac{U}{\sqrt{z_{e}^{2}+k^{2} U^{2}}} z_{e}^{2}-k_{2}\left(v_{e}-v_{v}\right)^{2}-k_{3} q_{e}^{2}\right. \\
& \left.+q_{e} g_{q}\left(\tilde{\varpi}_{0}^{T} \boldsymbol{\xi}_{0}+\tilde{\varpi}_{1}^{T} \boldsymbol{\xi}_{1} A+\hat{E} \operatorname{sgn}\left(q_{e}\right)-\epsilon_{01}\right)\right\} \\
& <\infty
\end{aligned}
$$

According to Barbalat lemma, it can be concluded that $\lim _{t \rightarrow \infty} P(t)=0$, namely $x_{e}=0, z_{e}=0$, and $v_{e}=0$. In summary, the model-free adaptive control laws composed of (10), (21) and (22) ensure that all the bottom following errors in (7) tend to zero in spite of fully unknown hydrodynamic coefficients and environmental disturbances. This concludes the proof of theorem 2 .

Remark 5 It can be seen from (21) that the model-free adaptive control law relaxes the dependence on the accurate AUV hydrodynamic coefficients, environmental disturbance model and high-order derivatives. However, it generates an unsaturated rudder command. As mentioned in remark 2, the inherent control surface saturation will lead to control input truncation even affect the system stability. The following is an improved model-free adaptive control law, which guarantees the asymptotic stability of robust bottom following control system in the presence of delayed and saturated control surfaces. 


\section{Model-free Adaptive Controller with Delayed and Saturat- ed Control Surfaces}

Due to the existence of inherent saturation, the saturated adaptive control law for the control surface deflection command of a flight-style AUV has to be updated as follows:

$$
\delta_{c}=\operatorname{sat}\left(\delta_{c a}\right)=\left\{\begin{array}{lr}
\delta_{c, \max }, & \delta_{c a}>\delta_{s, \max } \\
\delta_{c a}, & \left|\delta_{c a}\right| \leq \delta_{s, \max } \\
-\delta_{c, \max }, & \delta_{c a}<-\delta_{s, \max }
\end{array}\right.
$$

where $\delta_{c a}$ is the stern control surface deflection calculated by the adaptive controller and is usually unsaturated.

Define the difference between the unsaturated output and the constrained one as,

$$
\vartheta=\delta_{c}-\delta_{c a}
$$

To address the above difference between the unsaturated and saturated outputs and avoid the potential unstable control behavior, an additional fuzzy compensator is inserted into the unsaturated control law, to compensate the control truncation, as shown in Fig. 5. The following are main results of this model-free saturated adaptive controller.

According to Lemma 1, there exists an optimal fuzzy compensation system such that,

$$
\vartheta=\varpi_{2}^{* \top} \boldsymbol{\xi}_{2}+\epsilon_{2}
$$

where $\epsilon_{2}$ is the compensation error.

Assumption 5 [21] The compensation error $\epsilon_{2}$ satisfies $\left|\epsilon_{01}\right|+\left|\epsilon_{2}\right| \leq E$ with an unknown upper bound $E>0$.

Theorem 3 Consider a flight-style AUV with the kinematics equations in (1) and the dynamics equations in (3), and assume that the surge velocity $u$ remains constant. Let the virtual control law $\dot{s}$ be updated by (10) and the stern control surface deflection command $\delta_{c}$ be updated by,

$$
\delta_{c}=\operatorname{sat}\left(\hat{\varpi}_{0}^{T} \boldsymbol{\xi}_{0}+\hat{\varpi}_{1}^{T} \boldsymbol{\xi}_{1} A+\hat{E} \operatorname{sgn}\left(q_{e}\right)-\hat{\varpi}_{2}^{T} \boldsymbol{\xi}_{2}\right)
$$

where $\hat{\varpi}_{0}, \hat{\varpi}_{1}, \hat{\varpi}_{2}$ and $\hat{E}$ are adaptive estimations of $\varpi_{0}^{*}$, $\varpi_{1}^{*}, \varpi_{2}^{*}$ and $E$, respectively. They are governed by,

$$
\begin{aligned}
\dot{\hat{\varpi}}_{0} & =\eta_{0}{ }^{-1} q_{e} \boldsymbol{\xi}_{0} \\
\dot{\hat{\varpi}}_{1} & =\eta_{1}{ }^{-1} q_{e} \boldsymbol{\xi}_{1} A \\
\dot{\hat{E}} & =\eta_{2}{ }^{-1}\left|q_{e}\right| \\
\dot{\boldsymbol{\varpi}}_{2} & =-\eta_{3}{ }^{-1} q_{e} \boldsymbol{\xi}_{2}
\end{aligned}
$$

with $\eta_{0}>0, \eta_{1}>0, \eta_{2}>0$ and $\eta_{3}>0$. Then, the equilibrium point $\left(x_{e}, z_{e}, v_{e}\right)=(0,0,0)$ of the entire bottom following control system is stable.

Proof Recalling $\delta_{c}$ in (33) yields,

$$
\begin{aligned}
\delta_{c} & =\operatorname{sat}\left(\hat{\varpi}_{0}^{T} \boldsymbol{\xi}_{0}+\hat{\varpi}_{1}^{T} \boldsymbol{\xi}_{1} A+\hat{E} \operatorname{sgn}\left(q_{e}\right)-\hat{\varpi}_{2}^{T} \boldsymbol{\xi}_{2}\right) \\
& =\hat{\varpi}_{0}^{T} \boldsymbol{\xi}_{0}+\hat{\varpi}_{1}^{T} \boldsymbol{\xi}_{1} A+\hat{E} \operatorname{sgn}\left(q_{e}\right)-\hat{\varpi}_{2}^{T} \boldsymbol{\xi}_{2}+\vartheta \\
& =\hat{\varpi}_{0}^{T} \boldsymbol{\xi}_{0}+\hat{\varpi}_{1}^{T} \boldsymbol{\xi}_{1} A+\hat{E} \operatorname{sgn}\left(q_{e}\right)-\tilde{\varpi}_{2}^{T} \boldsymbol{\xi}_{2}+\epsilon_{2}
\end{aligned}
$$

with $\tilde{\varpi}_{2}=\hat{\varpi}_{2}-\varpi_{2}^{*}$.
Substituting (20) and (35) into (4) yields,

$$
\begin{aligned}
\dot{q}-\dot{q}_{d}= & -k_{3} q_{e}-v_{e}+v_{v} \\
& +g_{q}\left(\tilde{\varpi}_{0}^{T} \boldsymbol{\xi}_{0}+\tilde{\varpi}_{1}^{T} \boldsymbol{\xi}_{1} A-\tilde{\varpi}_{0}^{T} \boldsymbol{\xi}_{0}\right) \\
& +g_{q}\left(\hat{E} \operatorname{sgn}\left(q_{e}\right)-\epsilon_{01}+\epsilon_{2}\right)
\end{aligned}
$$

Define the final radically unbounded and positive definite Lyapunov candidate function $V_{3}$ as follows:

$$
V_{3}=V_{2}-\frac{1}{2} g_{q} \eta_{3} \tilde{\varpi}_{2}^{T} \tilde{\varpi}_{2}
$$

Resorting to the error dynamics (8), (36) and (16), the derivative of $V_{3}$ is,

$$
\begin{aligned}
& \dot{V}_{3}=-k_{1} x_{e}^{2}-\frac{U}{\sqrt{z_{e}^{2}+k^{2} U^{2}}} z_{e}^{2}-k_{2}\left(v_{e}-v_{v}\right)^{2}-k_{3} q_{e}^{2} \\
& +q_{e} g_{q}\left(\tilde{\varpi}_{0}^{T} \boldsymbol{\xi}_{0}+\tilde{\varpi}_{1}^{T} \boldsymbol{\xi}_{1} A-\tilde{\varpi}_{2}^{T} \boldsymbol{\xi}_{2}\right) \\
& -g_{q} \eta_{0} \tilde{\varpi}_{0}^{T} \dot{\hat{\varpi}}_{0}-g_{q} \eta_{1} \tilde{\varpi}_{1}^{T} \dot{\hat{\varpi}}_{1}-g_{q} \eta_{3} \tilde{\varpi}_{2}^{T} \dot{\hat{\varpi}}_{2} \\
& +q_{e} g_{q}\left(\hat{E} \operatorname{sgn}\left(q_{e}\right)-\epsilon_{01}+\epsilon_{2}\right)-g_{q} \eta_{2} \dot{\hat{E}}(\hat{E}-E) \\
& =-k_{1} x_{e}^{2}-\frac{U}{\sqrt{z_{e}^{2}+k^{2} U^{2}}} z_{e}^{2}-k_{2}\left(v_{e}-v_{v}\right)^{2}-k_{3} q_{e}^{2} \\
& +g_{q} \tilde{\boldsymbol{\varpi}}_{0}^{T}\left(q_{e} \boldsymbol{\xi}_{0}-\eta_{0} \dot{\hat{\boldsymbol{\omega}}}_{0}\right)+g_{q} \tilde{\boldsymbol{\varpi}}_{1}^{T}\left(q_{e} \boldsymbol{\xi}_{1} A-\eta_{1} \dot{\hat{\boldsymbol{\varpi}}}_{1}\right) \\
& +g_{q} \tilde{\boldsymbol{\varpi}}_{2}^{T}\left(q_{e} \boldsymbol{\xi}_{2}+\eta_{3} \dot{\hat{\boldsymbol{\varpi}}}_{2}\right)+g_{q} \hat{E}\left(\left|q_{e}\right|-\eta_{2} \dot{\hat{E}}\right) \\
& -q_{e} g_{q}\left(\epsilon_{01}-\epsilon_{2}\right)+g_{q} E \eta_{2} \dot{\hat{E}} \\
& =-k_{1} x_{e}^{2}-z_{e}^{2} \frac{U}{\sqrt{z_{e}^{2}+k^{2} U^{2}}}-k_{2}\left(v_{e}-v_{v}\right)^{2}-k_{3} q_{e}^{2} \\
& +g_{q}\left(E\left|q_{e}\right|-q_{e}\left(\epsilon_{01}-\epsilon_{2}\right)\right) \\
& \leq-k_{1} x_{e}^{2}-z_{e}^{2} \frac{U}{\sqrt{z_{e}^{2}+k^{2} U^{2}}}-k_{2}\left(v_{e}-v_{v}\right)^{2}-k_{3} q_{e}^{2}
\end{aligned}
$$

Similar to the proof of theorem 2, it can be obtained that $\lim _{t \rightarrow \infty}\left(x_{e}(t), z_{e}(t), v_{e}(t)\right)=(0,0,0)$. This concludes the proof of theorem 3 .

\section{NUMERICAL SimULATION}

In order to illustrate the effectiveness of the designed robust adaptive bottom following controller, two kinds of comparative numerical simulations are conducted by using the REMUS AUV model [39]. One is the comparative analysis between the model-free and model-based controllers, and the other is the robust analysis of the designed saturated adaptive controller in different initial states. The REMUS AUV hydrodynamic coefficients used in the simulation are listed in Table I.

In the simulation, assume the REMUS AUV is exposed to the following unknown time-varying environmental disturbances:

$$
\begin{aligned}
\tau_{E u} & =0.15\left(m-X_{\dot{u}}\right) d(t) \\
\tau_{E w} & =0.15\left(m-Z_{\dot{w}}\right) d(t) \\
\tau_{E q} & =0.1\left(I_{y y}-M_{\dot{q}}\right) d(t)
\end{aligned}
$$

with $d(t)=1+0.1 \sin (0.25 t)$, and the desired bottom following path is parameterized by,

$$
\begin{aligned}
& x_{d}(s)=1.5 s \\
& z_{d}(s)=20+1.5 \sin (0.1 s)
\end{aligned}
$$




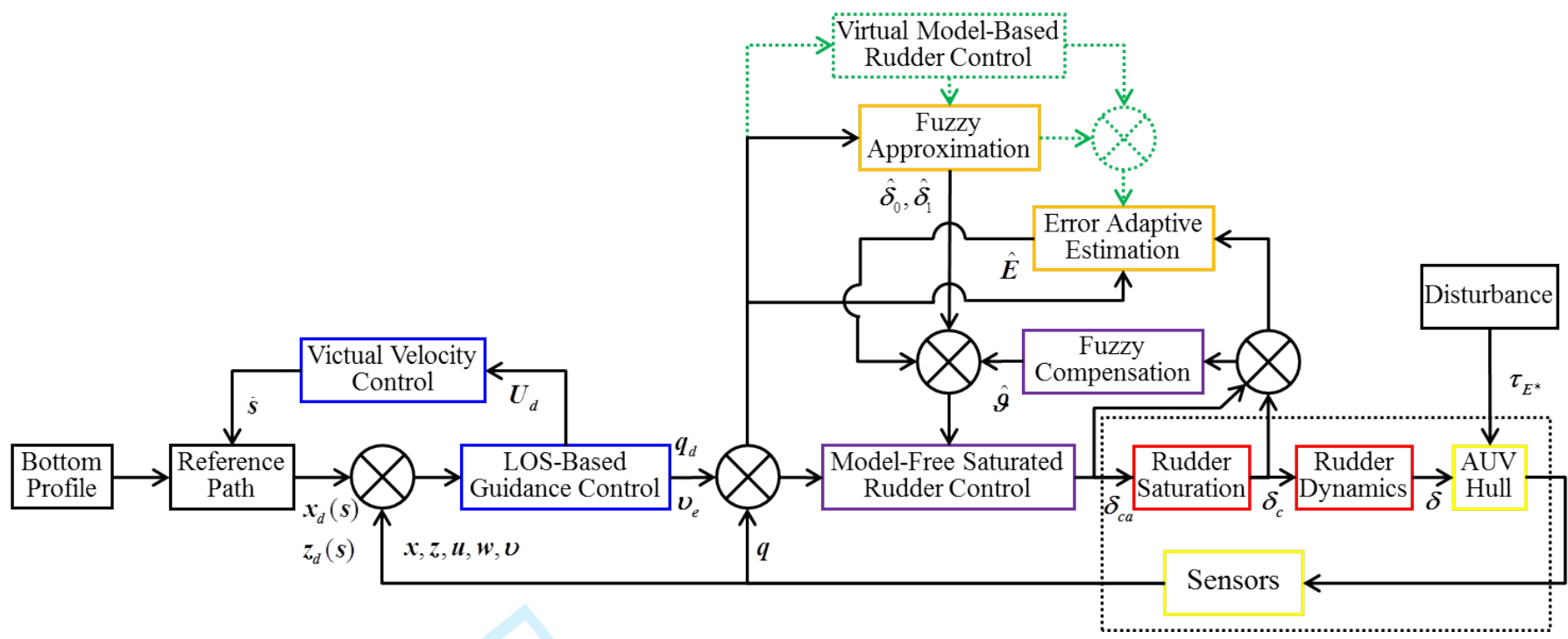

Fig. 5. Block diagram of the model-free saturated bottom following control system

where the initial value of $s$ is $s(0)=0$.

The parameters of the designed model-free saturated adaptive bottom following controller are chosen as follows: $k_{1}=$ $1.5, k_{2}=5, k_{3}=12.5, k=13.5, \eta_{0}=110, \eta_{1}=0.01$, $\eta_{2}=1000, \eta_{3}=10.75$.

\section{A. Case I - Comparative Performance Analysis of the Model- free and Model-based Controllers}

To demonstrate the superiority of the designed robust bottom following controller, the model-based backsteppping control, the model-free unsaturated controller and the model-free saturated controller are tested, respectively. The AUV initial position and posture are $[x(0), z(0), \theta(0)]=[-4 \mathrm{~m}, 15 \mathrm{~m}, 0 \mathrm{rad}]$, and its initial velocities are $[u(0), w(0), q(0)]=[1.25 \mathrm{~m} / \mathrm{s}, 0 \mathrm{~m} / \mathrm{s}, 0 \mathrm{rad} / \mathrm{s}]$.

TABLE I

REMUS HYDRODYNAMIC COEFFICIENTS

\begin{tabular}{ccc}
\hline Coefficient & Value & Unit \\
\hline$m$ & 30.48 & $\mathrm{~kg}$ \\
$W$ & 299 & $\mathrm{~N}$ \\
$B$ & 306 & $\mathrm{~N}$ \\
$I_{y y}$ & 3.45 & $\mathrm{~N} \cdot \mathrm{kg} \cdot \mathrm{m}^{2}$ \\
$z_{G}$ & 0.0196 & $\mathrm{~m}$ \\
$M_{\dot{q}}$ & -4.88 & $\mathrm{~kg} \cdot \mathrm{m}^{2} \cdot \mathrm{rad}^{-1}$ \\
$M_{\dot{w}}$ & -1.93 & $\mathrm{~kg} \cdot \mathrm{m}$ \\
$M_{w|w|}$ & 3.18 & $\mathrm{~kg}$ \\
$M_{q|q|}$ & -188 & $\mathrm{~kg} \cdot \mathrm{m}^{2} \cdot \mathrm{rad}^{-2}$ \\
$M_{u q}$ & -2 & $\mathrm{~kg} \cdot \mathrm{m} \cdot \mathrm{rad}^{-1}$ \\
$M_{u w}$ & 24 & $\mathrm{~kg}$ \\
$M_{u u \delta}$ & -6.15 & $\mathrm{~kg} \cdot \mathrm{rad}^{-1}$ \\
$Z_{\dot{w}}$ & -35.5 & $\mathrm{~kg}$ \\
$Z_{w|w|}$ & -131 & $\mathrm{~kg} \cdot \mathrm{m}^{-1}$ \\
$Z_{q|q|}$ & -0.632 & $\mathrm{~kg} \cdot \mathrm{m} \cdot \mathrm{rad}^{-2}$ \\
$Z_{u w}$ & -28.6 & $\mathrm{~kg} \cdot \mathrm{m}^{-1}$ \\
$Z_{u q}$ & -5.22 & $\mathrm{~kg} \cdot \mathrm{rad}^{-1}$ \\
$\delta_{c, \max }$ & 30 & $\circ$ \\
$\lambda$ & 0.1 & $\mathrm{~s}$ \\
\hline & &
\end{tabular}

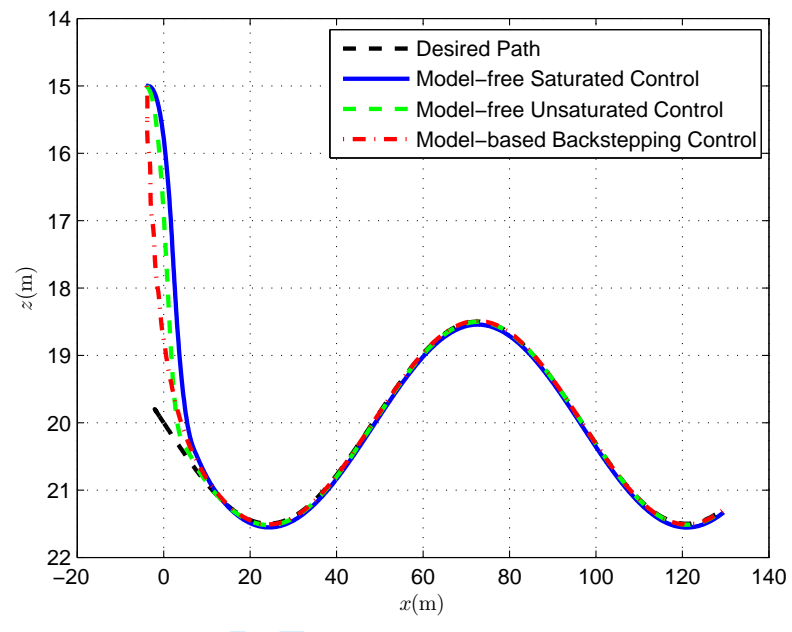

Fig. 6. Bottom following paths of three different controllers

Fig. 6 shows the bottom following paths of three different controllers, where the black dashed line is the desired bottom following path, and the other three colorful lines represent the simulated paths of the REMUS AUV. The corresponding errors including the depth error and the orientation angle error are depicted in Fig. 7. Although all of them quickly converge to the desired path, the model-based backsteppping controller leads to the shaking angle error, which likely results from the first-order and second-order derivative calculation. Obviously, the pitch angular velocity also suffers from the similar shaking behavior in Fig. 8. This shaking behavior occurs in the first 5 seconds because the error between the AUV and the path is relative large and the corresponding control law is such updated that the AUV can quickly converges to the desired path. 

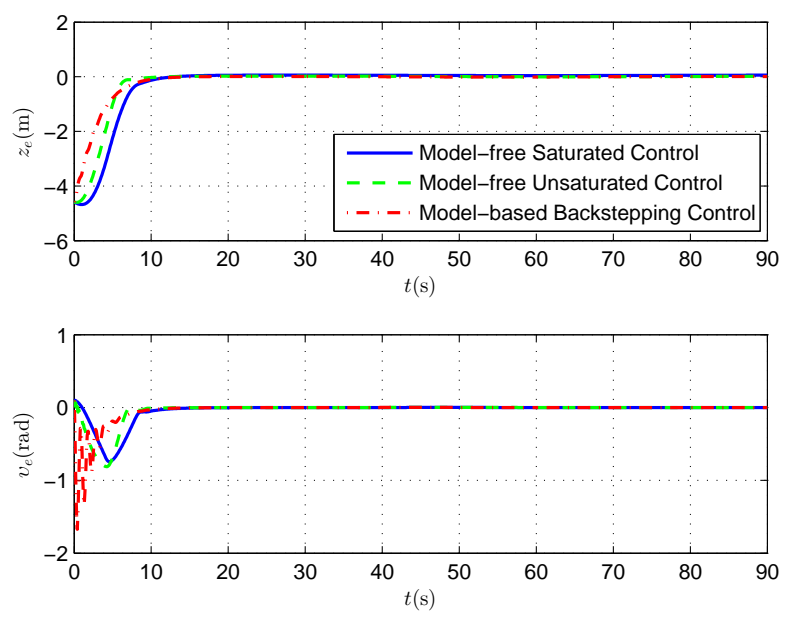

Fig. 7. Bottom following errors of three different controllers

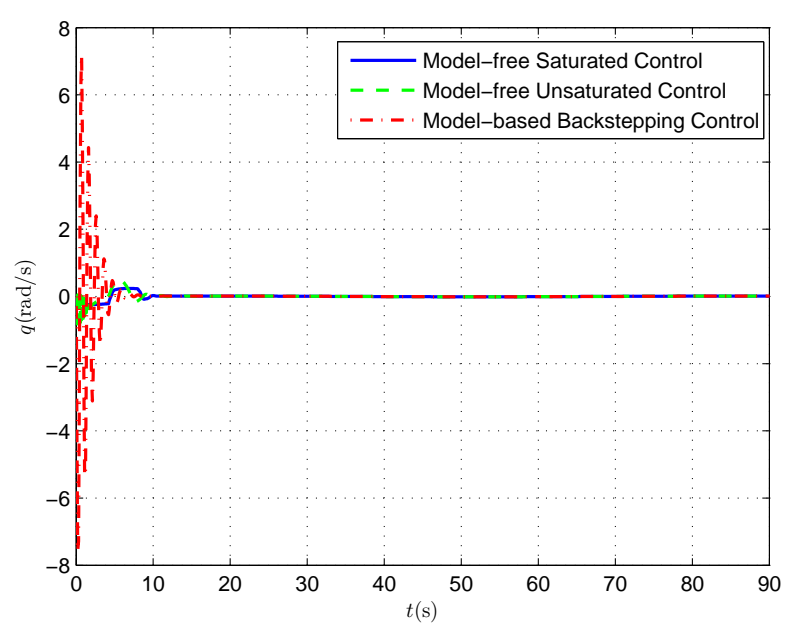

Fig. 8. Pitch angular velocities of three different bottom following controllers

As shown in Fig. 9, three stern control surface deflection commands are totally different. Note that the control surface deflection of the model-based backsteppping controller is divided by 200 in order to clearly show three command curves of robust bottom following control in one figure. Obviously, the model-based backsteppping controller generates a shaking and unsaturated stern control surface deflection curve in the first 5 seconds. Although the command of the model-free unsaturated controller does not shake, it is twice outside of the permitted range in the first 10 seconds. Therefore, the above two controllers cannot be directly applied to the actual AUV control system. Only the command of the model-free saturated controller has no shaking transition and well settles into the saturated control surface.

From comparative analysis of three different controllers, it can be concluded that the saturated adaptive controller without a prior knowledge on hydrodynamic coefficients and

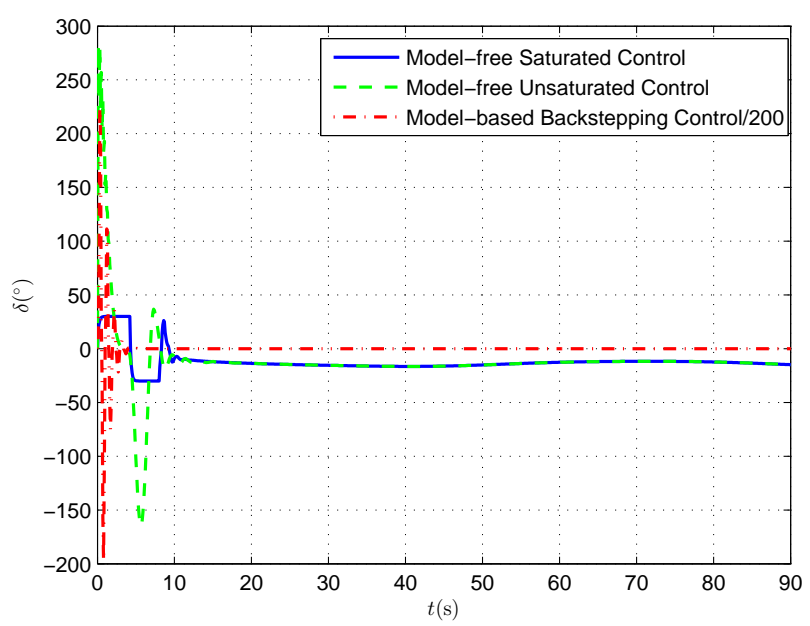

Fig. 9. Control commands of three different bottom following controllers

environmental disturbances avoids the shaking behavior of the model-based backstepping controller and guarantees the robust bottom following performance in spite of the saturated control surface deflection command.

\section{B. Case II - Robust Analysis of the Delayed and Saturated Controller in Different Initial States}

To further demonstrate the performance of the designed model-free saturated bottom following controller, four different REMUS initial positions and postures are considered in Table II and all the initial velocities are $[u(0), w(0), q(0)]=$ $[1.25 \mathrm{~m} / \mathrm{s}, 0 \mathrm{~m} / \mathrm{s}, 0 \mathrm{rad} / \mathrm{s}]$. Note that all the control parameters are the same for these four states.

The corresponding bottom following response paths under the saturated control laws are described in Fig. 10, where the red dashed line is the desired tracking path that has a height of $1.5 \mathrm{~m}$ over a undulating bottom profile, and the other four colorful lines represent the simulated paths of the REMUS AUV. From Fig. 10, it can be observed that the REMUS AUV converges to the desired path and moves along this path regardless of the initial position and posture.

For state 1 , the position and orientation errors in the entire following period are drawn in Fig. 11, which shows all of them stabilize at zero after 14.8 seconds. It can be concluded that the designed bottom following controller approximates the optimal control law and guarantees that all the errors including the position error and the orientation error are bounded and tend to zero by using the robust fuzzy adaptive controller without a

TABLE II

DIFFERENT INITIAL POSITIONS AND POSTURES

\begin{tabular}{cc}
\hline State & Value \\
\hline 1 & {$[x(0), z(0), \theta(0)]=[-4 \mathrm{~m}, 15 \mathrm{~m}, 0 \mathrm{rad}]$} \\
2 & {$[x(0), z(0), \theta(0)]=[-12 \mathrm{~m}, 16 \mathrm{~m}, 0.15 \mathrm{rad}]$} \\
3 & {$[x(0), z(0), \theta(0)]=[8 \mathrm{~m}, 17 \mathrm{~m}, 0 \mathrm{rad}]$} \\
4 & {$[x(0), z(0), \theta(0)]=[6 \mathrm{~m}, 18 \mathrm{~m},-0.15 \mathrm{rad}]$} \\
\hline
\end{tabular}




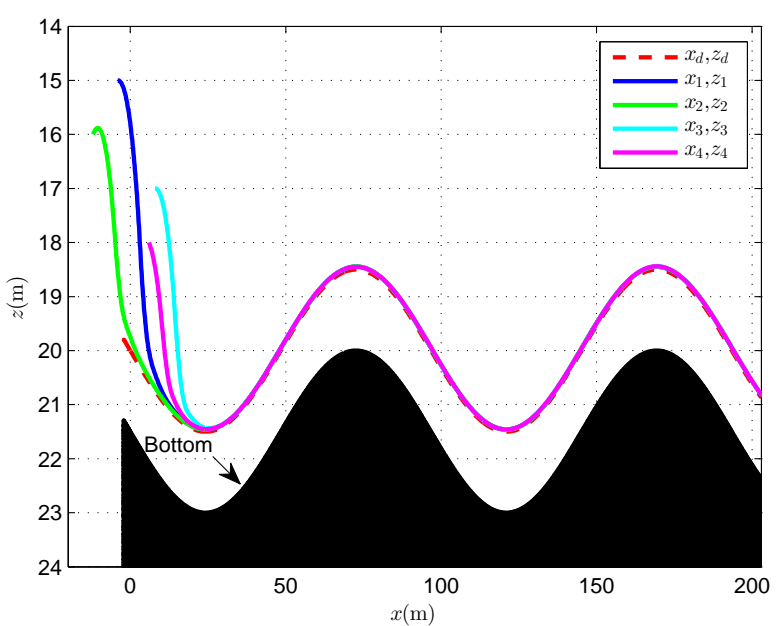

Fig. 10. Bottom following performance in different initial states
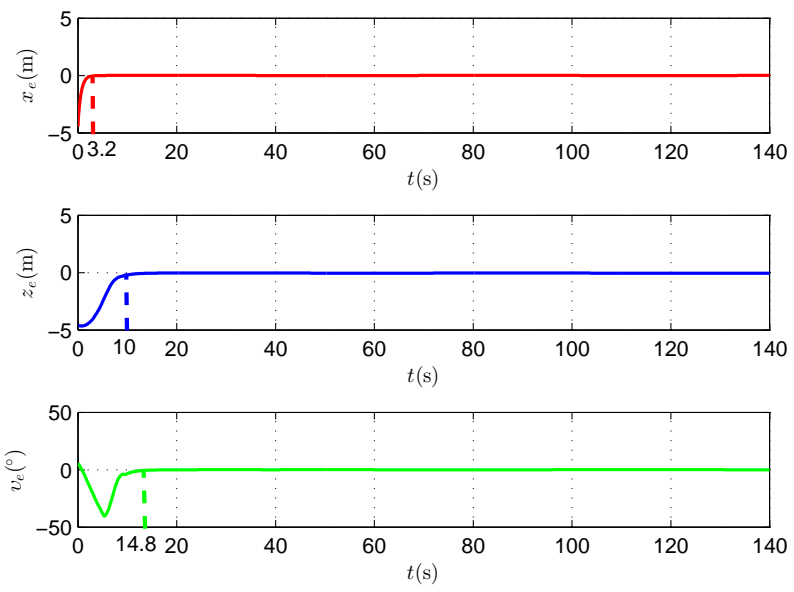

Fig. 11. Position and orientation errors

priori knowledge on the AUV hydrodynamic coefficients and environmental disturbances.

Fig. 12 gives the simulated stern control surface deflection and the deflection command with respect to time. Obviously, the simulated stern control surface deflection and deflection command changes within the permitted range. In addition, there exist differences between them at the same instant due to the time delay. Especially, the time delay leads to a smoother transition of the stern control surface deflection acting on the AUV.

From analysis of the saturated adaptive controller in different initial states, it can be concluded that the designed saturated fuzzy controller has a similar robust and adaptive performance regardless of the AUV initial states. Combining case A with case B, the designed model-free saturated fuzzy controller is proved to be qualified for the robust bottom following control mission of a flight-style AUV.

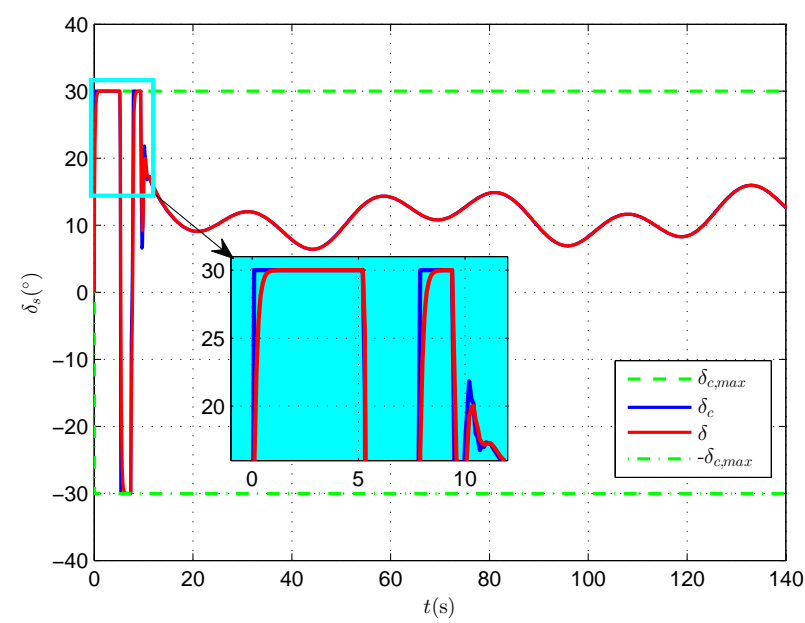

Fig. 12. Stern control surface deflection versus deflection command

\section{CONClusion}

In this paper, a novel model-free robust bottom following controller for a flight-style AUV with both delayed and saturated bottom is presented with theoretic and numerical analysis. First, a model-based second-order backstepping controller by utilizing guidance errors is designed for the second-order nonlinear dynamics while considering the timedelayed dynamics of rudders, which also plays a benchmark for robust adaptive controllers and meanwhile provides an unified control architecture for the robust bottom following problem; Second, inspired by the benchmark control architecture, a fuzzy approximator-based model-free control law is proposed to online approximate the unknown part of the ideal backstepping control architecture, which overcomes the potential shaking control behavior resulted by the model-based high-order derivative calculation. In addition, the approximation error is online estimated by the adaptive estimation technology, in order to drive all the position and orientation errors of robust bottom following control converging to zero; Third, an additional adaptive fuzzy control law is resorted to compensate control truncation between the unsaturated and saturation inputs and tackle the potential unstable control behavior. Asymptotic stability of the entire bottom following control system is synthesized by adopting Lyapunov theory and Barbalat lamma. Finally, comparative numerical results with two case studies illustrate the adaptive and robust performance of the designed delayed and saturated bottom following control system. Future work will be extended to the threedimensional bottom following problem of a flight-style AUV by using two pairs of delayed and saturated control surfaces.

\section{REFERENCES}

[1] H. Singh, A. Can, R. Eustice, S. Lerner, N. Mcphee, and C. Roman, "Seabed auv offers new platform for high-resolution imaging," Eos Transactions American Geophysical Union, vol. 85, no. 31, pp. 289296, 2004.

[2] F. Zhang, G. Marani, R. N. Smith, and H. T. Choi, "Future trends in marine robotics," IEEE Robotics \& Automation Magazine, vol. 22, no. 1, pp. 14-122, 2015. 
[3] Z. Ju and H. Liu, "Recognizing hand grasp and manipulation through empirical copula," International Journal of Social Robotics, vol. 2, no. 3 , pp. 321-328, 2010.

[4] X. Xiang, C. Yu, Z. Niu, and Q. Zhang, "Subsea cable tracking by autonomous underwater vehicle with magnetic sensing guidance," Sensors, vol. 16, no. 8, p. 1335, 2016.

[5] Z. Peng, J. Wang, and D. Wang, "Containment maneuvering of marine surface vehicles with multiple parameterized paths via spatial-temporal decoupling," IEEE/ASME Transactions on Mechatronics, vol. 22, no. 2, pp. 1026-1036, 2017.

[6] K. Tanakitkorn, P. A. Wilson, S. R. Turnock, and A. B. Phillips, "Depth control for an over-actuated, hover-capable autonomous underwater vehicle with experimental verification," Mechatronics, vol. 41, pp. 6781, 2017.

[7] P. Encarnacao and A. Pascoal, "3d path following for autonomous underwater vehicle," in Proceedings of the 39th IEEE Conference on Decision and Control, pp. 2977-2982, 2000.

[8] L. Lapierre and D. Soetanto, "Nonlinear path-following control of an auv," Ocean Engineering, vol. 34, no. 11-12, pp. 1734-1744, 2007.

[9] M. Breivik and T. I. Fossen, "Principles of guidance-based path following in $2 \mathrm{~d}$ and 3d," in Proceedings of the 44th IEEE Conference on Decision and Control, and the European Control Conference, pp. 627634, 2005

[10] J. Yu, J. Liu, Z. Wu, and H. Fang, "Depth control of a bioinspired robotic dolphin based on sliding-mode fuzzy control method," IEEE Transactions on Industrial Electronics, vol. 65, no. 3, pp. 2429-2438, 2018.

[11] T. I. Fossen and A. M. Lekkas, "Direct and indirect adaptive integral line-of-sight path-following controllers for marine craft exposed to ocean currents," International Journal of Adaptive Control and Signal Processing, vol. 31, no. 4, pp. 445-463, 2017.

[12] K. Y. Pettersen, F. Mazenc, and H. Nijmeijer, "Global uniform asymptotic stabilization of an underactuated surface vessel: experimental results," IEEE Transactions on Control Systems Technology, vol. 12, no. 6, pp. 891-903, 2004.

[13] K. D. Do, "Global robust adaptive path-tracking control of underactuated ships under stochastic disturbances," Ocean Engineering, vol. 111, pp. 267-278, 2016.

[14] J. Gao, A. A. Proctor, Y. Shi, and C. Bradley, "Hierarchical model predictive image-based visual servoing of underwater vehicles with adaptive neural network dynamic control," IEEE Transactions on Cybernetics, vol. 46, no. 10, pp. 2323-2334, 2016.

[15] X. Xiang, C. Yu, L. Lapierre, J. Zhang, and Q. Zhang, "Survey on fuzzy-logic-based guidance and control of marine surface vehicles and underwater vehicles," International Journal of Fuzzy Systems, 2017. doi: 10.1007/s40815-017-0401-3.

[16] J.-Y. Park, B.-H. Cho, and J.-K. Lee, "Trajectory-tracking control of underwater inspection robot for nuclear reactor internals using time delay control," Nuclear Engineering and Design, vol. 239, no. 11, pp. $2543-2550,2009$.

[17] N. Fischer, D. Hughes, P. Walters, E. M. Schwartz, and W. E. Dixon, "Nonlinear rise-based control of an autonomous underwater vehicle," IEEE Transactions on Robotics, vol. 30, no. 4, pp. 845-852, 2014.

[18] C. P. Bechlioulis, G. C. Karras, S. Heshmati-Alamdari, and K. J. Kyriakopoulos, "Trajectory tracking with prescribed performance for underactuated underwater vehicles under model uncertainties and external disturbances," IEEE Transactions on Control Systems Technology, vol. 25, no. 2, pp. 429-440, 2017.

[19] Z. Ju and H. Liu, "A unified fuzzy framework for human-hand motion recognition," IEEE Transactions on Fuzzy Systems, vol. 19, no. 5, pp. 901-913, 2011.

[20] X. Xiang, C. Yu, and Q. Zhang, "Robust fuzzy 3d path following for autonomous underwater vehicle subject to uncertainties," Computers \& Operations Research, vol. 84, pp. 165-177, 2017.

[21] N. Wang, M. J. Er, J.-C. Sun, and Y.-C. Liu, "Adaptive robust online constructive fuzzy control of a complex surface vehicle system," IEEE Transactions on Cybernetics, vol. 46, no. 7, pp. 1511-1523, 2016.

[22] R. Cui, C. Yang, Y. Li, and S. Sharma, "Adaptive neural network control of auvs with control input nonlinearities using reinforcement learning," IEEE Transactions on Systems, Man, and Cybernetics: Systems, vol. 47, no. 6, pp. 1019-1029, 2017.

[23] Z. Peng, J. Wang, and D. Wang, "Distributed containment maneuvering of multiple marine vessels via neurodynamics-based output feedback," IEEE Transactions on Industrial Electronics, vol. 64, no. 5, pp. 38313839, 2017.

[24] W. He, H. Huang, and S. S. Ge, "Adaptive neural network control of a robotic manipulator with time-varying output constraints," IEEE Transactions on Cybernetics, vol. 47, no. 10, pp. 3136-3147, 2017.

[25] K. Shojaei, "Neural network formation control of underactuated autonomous underwater vehicles with saturating actuators," Neurocomputing, vol. 194, pp. 372-384, 2016.

[26] Y. Shen and J. Wang, "Robustness of global exponential stability of nonlinear systems with random disturbances and time delays," IEEE Transactions on Systems, Man, and Cybernetics: Systems, vol. 46, no. 9, pp. 1157-1166, 2016.

[27] M. S. Naik and S. N. Singh, "State-dependent riccati equation-based robust dive plane control of auv with control constraints," Ocean Engineering, vol. 34, no. 11, pp. 1711-1723, 2007.

[28] C. Shen, Y. Shi, and B. Buckham, "Integrated path planning and tracking control of an auv: A unified receding horizon optimization approach," IEEE/ASME Transactions on Mechatronics, vol. 22, no. 3, pp. 1163$1173,2017$.

[29] K. Shojaei, "Neural adaptive robust control of underactuated marine surface vehicles with input saturation," Applied Ocean Research, vol. 53, pp. 267-278, 2015.

[30] C. Yu, X. Xiang, Q. Zhang, and G. Xu, "Adaptive fuzzy trajectory tracking control of an under-actuated autonomous underwater vehicle subject to actuator saturation," International Journal of Fuzzy Systems, 2017. doi: 10.1007/s40815-017-0396-9.

[31] Z. Zheng, C. Jin, M. Zhu, and K. Sun, "Trajectory tracking control for a marine surface vessel with asymmetric saturation actuators," Robotics \& Autonomous Systems, vol. 97, pp. 83-91, 2017.

[32] T. Liu, W. Zhang, and D. Gu, "Analytical design of two-degree-offreedom control scheme for open-loop unstable processes with time delay," Journal of Process Control, vol. 15, no. 5, pp. 559-572, 2005.

[33] Y. Qu, H. Xu, W. Yu, H. Feng, and X. Han, "Inverse optimal controller for speed-varying path following of marine crafts with actuator dynamics," Journal of Marine Science \& Application, vol. 16, no. 2, pp. 225-236, 2017.

[34] W. Caharija, K. Y. Pettersen, M. Bibuli, P. Calado, E. Zereik, J. Braga, J. T. Gravdahl, A. J. Sorensen, M. Milovanovic, and G. Bruzzone, "Integral line-of-sight guidance and control of underactuated marine vehicles: Theory, simulations, and experiments," IEEE Transactions on Control Systems Technology, vol. 24, no. 5, pp. 1623-1642, 2016.

[35] A. M. Lekkas and T. I. Fossen, "Integral los path following for curved paths based on a monotone cubic hermite spline parametrization," IEEE Transactions on Control Systems Technology, vol. 22, no. 6, pp. $2287-$ 2301, 2014.

[36] X. Xiang, C. Liu, H. Su, and Q. Zhang, "On decentralized adaptive fullorder sliding mode control of multiple uavs," ISA Transactions, vol. 71, pp. 196-205, 2017.

[37] L.-X. Wang and J. M. Mendel, "Fuzzy basis functions, universal approximation, and orthogonal least-squares learning," IEEE Transactions on Neural Networks, vol. 3, no. 5, pp. 807-814, 1992.

[38] C. P. Chen, Y.-J. Liu, and G.-X. Wen, "Fuzzy neural network-based adaptive control for a class of uncertain nonlinear stochastic systems," IEEE Transactions on Cybernetics, vol. 44, no. 5, pp. 583-593, 2014.

[39] T. Prestero, "Verification of a six-degree of freedom simulation model for the remus autonomous underwater vehicle," Master's thesis, Massachusetts Institute of Technology, 2001.

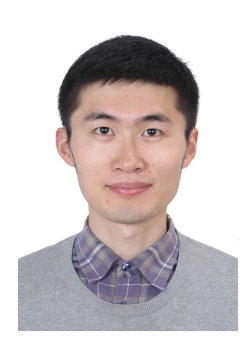

vehicles.
Caoyang Yu received the B.E. degree in marine engineering from the School of Naval Architecture and Ocean Engineering, Huazhong University of Science and Technology, Wuhan, China, in 2013, where he is currently working toward the Ph.D. degree in marine engineering.

Since September 2017, he has been a Visiting Research Student with the Faculty of Engineering and the Environment, University of Southampton, Southampton, UK. His current research interests include guidance and control for marine robotic 


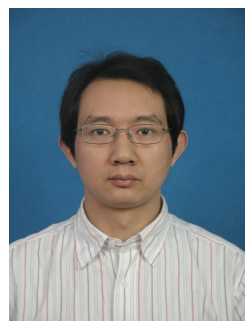

Xianbo Xiang received the B.E. and M.E. degrees in automatic control and marine engineering from Huazhong University of Science and Technology, Wuhan, China, in 2000 and 2003, respectively, and the $\mathrm{Ph} . \mathrm{D}$. degree in robotics from the University of Montpellier 2, Montpellier, France, in 2011.

$\mathrm{He}$ was a Lecturer at Huazhong University of Science and Technology. From September 2006 to December 2006, he was an EU Erasmus Mundus Visiting Scholar in the SpaceMaster Project. From February 2008 to March 2011, he was in the European Project FreeSubNet as an EC Marie Curie ESR Fellow at Laboratoire d' Informatique, de Robotique et de Microelectronique de Montpellier, CNRS UMR 5506, Montpellier, France. He is currently an Associate Professor in the School of Naval Architecture and Ocean Engineering, Huazhong University of Science and Technology. His research interests include robotics and marine vehicle systems.

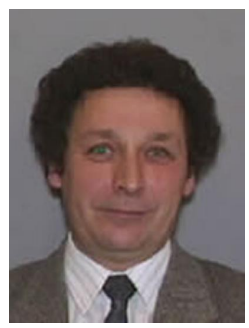

Philip A. Wilson received BSc(Hons) degree in mathematics for the University of Leicester, Leicester, UK, in 1968, and was subsequently awarded a DSc by the University of Leicester, in 2007.

Following work for Plessey Underwater systems he started in the University of Southampton in 1973 as a research fellow in the former Department of Aeronautics and Astronautics as part of the fledgling Ship Science group. From 2001, he has been Professor of Ship Dynamics within Engineering and the Environment at the University of Southampton. He is a founder member of the former Department of Ship Science and a Fellow of the Royal Institution of Naval Architects. Currently he is editor of the International Journal of Maritime Engineering and the Journal of Royal Institution of Naval Architects. He has published more than 250 academic papers. His research interests include seakeeping, ship manoeuvring, control of surface following ships, control of autonomous underwater vehicles, fluid dynamic flow in composite materials, collision avoidance techniques.

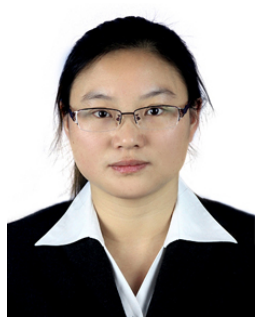

Qin Zhang received the B.S. degree in mechanical engineering from Northeast Petroleum University, Daqing, China, in 2000, the M.Sc. degree in marine engineering from Huazhong University of Science and Technology, Wuhan, China, in 2003, and the $\mathrm{Ph} . \mathrm{D}$. degree in robotics from the University of Montpellier 2, Montpellier, France, in 2011.

From January 2012 to March 2012, she was a Postdoctoral Fellow in the DEMAR Project, INRIA Sophia Antipolis. Since May 2012, she has been a JSPS Research Fellow affiliated with the Department of Mechanical Systems Engineering, Tokyo University of Agriculture and Technology. She is currently an Associate Professor in the State Key Laboratory of Digital Manufacturing, Equipment and Technology, Huazhong University of Science and Technology. Her research interests include control of biomechanical systems and robotic systems. 